\title{
EFECTOS DE VARIACIONES EN EL NIVEL DE DISCRIMINACIÓN DE LA AUTORIDAD, EN LA CONDUCTA DE TRANSGRESIÓN DE NORMAS ${ }^{I}$
}

\author{
EDWIN G. LUNA*, CHRISTIAN A. ZAMBRANO, FREDDY HIDALGO \\ UNIVERSIDAD DE NARIÑO - COLOMBIA \\ GRUPO DE INVESTIGACIÓN PSICOLOGÍA Y SALUD, LABORATORIO DE ANÁLISIS DE LA CONDUCTA
}

Recibido, agosto 30/2012

Concepto evaluación, junio 11/2013

Aceptado, agosto 14/2013
Referencia: Luna, E., Zambrano, C. \& Hidalgo, F. (2013). Efectos de variaciones en el nivel de discriminación de la autoridad en la conducta de transgresión de normas. Acta Colombiana de Psicología, 16 (2), 139-146.

Resumen

El objetivo de este estudio fue analizar los efectos diferenciales de las variaciones en el nivel de discriminación de la autoridad que administra la sanción, en la conducta de transgresión de una norma presentada de manera explícita. El trabajo presenta un diseño de investigación experimental con varios grupos de comparación y mediciones post. Se registró la frecuencia de transgresión en cada una de cuatro condiciones experimentales: RGe1: sin autoridad, RGe2: autoridad parcial señalada, RGe3: instrucciones de autoridad no señalada y, finalmente, RGe4: instrucciones de autoridad no señalada y observación de un caso de sanción. Los resultados muestran diferencias en las transgresiones a la norma ocurridas entre las diferentes condiciones experimentales, observándose más transgresiones en la condición sin autoridad, seguida de autoridad parcial señalada; en tercer lugar, la condición instrucciones de autoridad no señalada, y finalmente la condición instrucciones de autoridad no señalada y observación de un caso de sanción, en la que no se presentó transgresión. Al aplicar la prueba no paramétrica Kruskal-Wallis, estas diferencias muestran significancia estadística $(\mathrm{p}<0.001)$.

Palabras clave: conducta de transgresión, sanción, estudiantes universitarios.

\section{EFFECTS OF CHANGES IN THE LEVEL OF DIFFERENTIATION OF AUTHORITY ON THE BEHAVIOR OF RULE VIOLATION}

\begin{abstract}
The aim of this paper was to analyze the distinctive effects of variations in the level of differentiation of an authority that administers sanctions, on the behavior of transgressing a norm explicitly presented. This study used an experimental research design with multiple comparison groups and post measurements. The frequency of transgression in each of the four experimental conditions was recorded: RGe1: without authority, RGe2: partial explicit authority, RGe3: instructions of non-explicit authority, and finally RGe4: instructions of non-explicit authority and observation of a sanction case. Results show differences in the norm transgressions that occurred in the different experimental conditions. More transgressions were found in the condition without authority, followed by partial explicit authority, then in third place, the condition instructions of non-explicit authority and finally the condition of non-explicit instructions and observation of a sanction case, in which transgressions did not occur. In applying nonparametric Kruskal- Wallis test, these differences showed statistical significance $(\mathrm{p}<0.001)$

Keywords: transgression behavior, sanction, college students
\end{abstract}

\section{EFEITOS DE VARIAÇÓES NO NÍVEL DE DISCRIMINAÇÃO DA AUTORIDADE, NA CONDUTA DE TRANSGRESSÁO DE NORMAS}

\author{
Resumo
}

\begin{abstract}
O objetivo deste estudo foi analisar os efeitos diferenciais das variações no nível de discriminação da autoridade que administra a sanção na conduta de transgressão de analisar os efeitos diferenciais das variações no nível de discriminação da autoridade que administra a sanção, na conduta de transgressão de uma norma apresentada de maneira explícita. O trabalho apresenta um desenho de pesquisa experimental com vários grupos de comparação eI medições pós. Registrou-se a frequência de transgressão em cada uma das quatro condições experimentais: RGe1: sem autoridade, RGe2: autoridade parcial assinalada, RGe3: instruções de autoridade não
\end{abstract}

\footnotetext{
* Grupo de Investigación Piscología y Salud, Laboratorio de Análisis de la Conducta, Departamento de Psicología, Universidad de Nariño. lunedwingerardo@gmail.com

1 Los autores desean expresar un agradecimiento especial a Stephanie Martínez Muñoz por su colaboración en el trabajo de campo.
} 
assinalada e, finalmente, RGe4: instruções de autoridade não assinalada e observação de um caso de sanção. Os resultados mostram diferenças nas transgressões à norma ocorridas entre as diferentes condições experimentais, observando-se mais transgressões na condição sem autoridade, seguida de autoridade parcial assinalada em terceiro lugar, a condição instruções de autoridade não assinalada, e finalmente a condição instruções de autoridade não assinalada e observação de um caso de sanção, em que não houve transgressão. Ao aplicar o teste não paramétrico Kruskal-Wallis, estas diferenças mostram significância estatística ( $<<0.001)$.

Palavras chave: conduta de transgressão, sanção, estudantes universitários.

\section{INTRODUCCIÓN}

La conducta prosocial de algunos animales, facilitada por un medio de contacto ecológico que posibilita interacciones vinculadas a la supervivencia, supone interacciones desligadas de la reactividad biológica determinada por la filogénesis de la especie (Ribes, 2007). Este desligamiento funcional hace posible una reactividad que no está sujeta a las propiedades fisicoquímicas de los eventos, sino que implica responder a funciones de estímulo afectadas por relaciones de condicionalidad entre los eventos de la situación. No obstante, la funcionalidad de la interacción seguirá contextualizada por la situación en la que ocurren los eventos (Ribes y López, 1985).

A diferencia de lo anterior, la conducta social humana se caracteriza por la existencia de un medio de contacto convencional, que permite que la interacción entre dos o más personas trascienda funcionalmente la situación en espacio y tiempo, e incluso posibilite un responder autónomo respecto de eventos específicos (Ribes y López, 1985). Este medio de contacto convencional que es articulado por el lenguaje, no excluye, sino que se supraordina al medio de contacto ecológico y hace posible la convivencia, como un tipo de interacción que no difiere, sino que trasciende la supervivencia.

Ribes (2001) propone además que la conducta social se da exclusivamente en los humanos, y que "puede ser identificada sólo mediante la división del trabajo y los intercambios diferidos de bienes y servicios, todo ello posibilitado por la comunicación convencional o el lenguaje" (Ribes, 2001, p. 285). La relevancia de la división del trabajo y el intercambio para el análisis de la conducta social es sustentada en tanto estos dos fenómenos indican que la producción, como práctica social, se ha desligado de la situacionalidad en la que tiene lugar, para participar de una dimensión funcional convencional regulada por instituciones (Ribes, 2001).

Este nivel de autonomía funcional respecto a la situacionalidad espacio temporal, es una característica definitoria de la conducta social posibilitada por el lenguaje. Es decir, la conducta social tiene lugar en tanto se presenta la regulación sustitutiva, lo que supone que el comportamiento queda bajo el control de funciones de estímulo-respuesta con morfologías convencionales, las cuales no están de- terminadas por las características fisicoquímicas de los eventos (Ribes y López, 1985).

Antes de profundizar en las dimensiones de la conducta social, conviene mencionar que aunque el comportamiento humano es generalmente lingüístico y social, dada la naturaleza convencional del medio de contacto predominante en las interacciones de las personas, se propone la posibilidad de distinguir entre contingencias sociales y contingencias individuales (Ribes, et al., 2008). Concretamente, las contingencias sociales se presentan cuando el comportamiento de un individuo afecta directamente las circunstancias de otro u otros individuos, mientras que en las contingencias individuales, el comportamiento del individuo sólo afecta directamente al propio individuo que se comporta ( $c f$. Ribes y Rangel, 2002: web; $c f$. Ribes, Rangel, Magaña, López y Zaragoza, 2005: web; $c f$. Ribes et al., 2003: web; $c f$. Ribes et al., 2006: web; $c f$. Ribes et al., 2008: web).

Respecto al análisis de la conducta social, Ribes (2001) identifica tres dimensiones funcionales como marco conceptual para analizar la conducta social. En primer lugar se encuentran las contingencias de intercambio, que corresponden a la complementación como logro fundamental de estructuración social, y que incluyen el compartir y el apropiar como dos formas básicas de intercambio. Esta dimensión se considera básica en relación con las otras dos dimensiones (poder y de sanción), debido a que estas últimas sólo pueden presentarse en el contexto de asimetrías entre la producción, la apropiación y la inequidad en la distribución, todos estos fenómenos que se encuentran circunscritos a las interacciones de intercambio (Ribes, Rangel y López, 2008).

La dimensión funcional de intercambio es sobre la que se ha realizado el mayor número de estudios hasta el momento. Específicamente, se ha investigado sobre la elección entre contingencias sociales o individuales vinculadas a altruismo parcial, altruismo total y competencia (Ribes y Rangel, 2002; Ribes et al. 2005); sobre el efecto de "forzar" inicialmente a los participantes a participar de contingencias sociales, en la elección posterior entre estas últimas y las contingencias individuales (Ribes et al. 2003); aislamiento verbal de los participantes mientras resuelven tareas experimentales (Ribes, Rangel, Magaña, Ramírez y Valdés, 2005) y, finalmente, sobre diferentes consecuencias y formas de distribución al elegir entre contingencias individuales o sociales (Ribes et al. 2006; Ribes et al. 2008). 
Como elemento común en los resultados de estos trabajos se ha encontrado que en circunstancias de elección, las personas prefieren interactuar bajo contingencias individuales. Sin embargo, también se ha observado que forzar a participar de contingencias sociales que generan una retribución significativamente mayor en comparación con contingencias individuales, al igual que el establecimiento de acuerdos verbales previos a la situación de elección, facilita el cambio en la preferencia de elección hacia las contingencias sociales.

En cuanto a la dimensión funcional de poder, según la propuesta de Ribes, Rangel y López (2008), ésta se sustenta en fuentes como la riqueza, la fuerza y/o el conocimiento. Además, esta dimensión se puede presentar de dos maneras, que no necesariamente tienen relación de incompatibilidad o exclusión entre ellas: por acuerdo o convención, que supone dominación implícita, y/o por coacción, que implica dominación explícita.

Sobre las contingencias específicas que integran la dimensión funcional de poder, Ribes, Rangel y López (2008) y Ribes (2001), identifican las contingencias de prescripción, regulación, supervisión y administración. Estas modalidades de la dimensión de poder establecen consecuencias en relación con la conducta social y pueden estar presentes simultáneamente en una situación o presentarse de manera separada (Ribes, Rangel y López, 2008).

En relación con esta dimensión en concreto, se ha investigado sobre el efecto de variaciones en el nivel de autoridad (determinada por la inclusión de las contingencias que integran la dimensión funcional de poder) en el cumplimiento y la obediencia (Rangel, Ribes, Valdéz y Pulido, 2011; Rangel y Ribes, 2009). Estos estudios, que se han desarrollado con niños escolares, sugieren hasta el momento que la autoridad es más efectiva cuando el poder se basa en la fuerza, que cuando lo hace en la riqueza, y que la función de prescripción parece ser la más efectiva para generar conducta obediente.

Finalmente, la conducta social se integra también por una dimensión funcional de sanción, con logros de acotamiento o limitación. Esta dimensión se caracteriza por la restricción y límite de la conducta de los individuos enmarcados en instituciones sociales, que se concreta en normas formuladas de manera explícita o tácitamente conocidas, que estipulan consecuencias en el caso de que se juzgue su ajuste o desajuste a la misma (Ribes, Rangel y López, 2008).

La trasgresión de normas (comportarse contraviniendo una prescripción) puede sancionarse de manera negativa, o no hacerlo y configurarse así un caso de impunidad. La sanción que sigue a un caso de transgresión de normas es negativa, en tanto siempre implica la pérdida parcial o total de bienes, derechos o del acceso a los mismos. Algunos de los tipos más comunes de sanción negativa son la amonestación, la suspensión, la expulsión, la privación de libertad, la separación, la multa, la exención, la anulación, la compensación o indemnización, el cese y la privación de la vida, entre muchos otros que variarán de acuerdo a la clase de institución social que enmarque la interacción social en el que tiene lugar la sanción (Ribes, Rangel y López, 2008).

Aunque no se encuentran estudios experimentales que aborden la dimensión de sanción de manera separada respecto de las de intercambio y poder, en estudios de Carpio et al. (2008a); Carpio et al. (2008b), sobre los efectos de factores situacionales e interacciones lingüísticas en la conducta cooperativa, se encontró que la elección de contingencias colaborativas era más probable en la situación de interacciones lingüísticas que fomentaban la conducta cooperativa (al indicar que la conducta cooperativa es valorada positivamente por la sociedad), y consecuencias situacionales negativas para la conducta de no-cooperar (pérdida de puntos o aumento en el puntaje a acumular). Aunque el estudio no tiene como objeto la dimensión de sanción, evidentemente la incluye, permite observar su importante efecto sobre la conducta de los participantes sancionados por no cooperar.

En un estudio de Medina y Polanco (2009) se involucraron las tres dimensiones. En este trabajo se observó qué efecto tenía en la conducta de cooperación la variación de instrucciones sobre relaciones de intercambio, poder y sanción. Los resultados del estudio sugieren que en los participantes del estudio, la conducta de cooperar, no cooperar, fue influida por las instrucciones que señalan relaciones de sanción, coincidiendo en este punto con los estudios de Carpio et al., (2008a), y relaciones de poder, lo que no se observó para el caso de las instrucciones de relaciones de intercambio, que parecieron no tener un efecto contundente, pues en ellas hubo amplia variabilidad en la conducta de los participantes.

Por otro lado, aunque desde aproximaciones teóricas más afines a la propuesta skinneriana se han abordado algunos aspectos relacionados con las contingencias de sanción, estas aproximaciones han enfatizado en el estudio de variables situacionales relacionadas con el castigo y la evitación (e.g. Bouzas, Orduña y Palafox, 2006; Domjan, 2007), sin considerar - por lo menos no en toda su trascendencia- el cambio cualitativo en la dimensión funcional de la interacción que implica el medio de contacto convencional característico de la conducta social (Ribes y López, 1985; Ribes, Rangel y López, 2008).

Teniendo en cuenta los antecedentes con relación a la investigación sobre dimensiones sociales, este trabajo 
pretende aportar al estudio de la dimensión de poder, aun cuando se comprende que no es posible estudiar una de estas dimensiones de forma realmente independiente de las otras dos.

Específicamente, se pretende observar el efecto de variaciones en el nivel de discriminación de la autoridad, sobre la conducta de transgresión de una norma formulada de manera explícita. El tipo de transgresión a observar será el de no ajuste a la norma por comisión en un ámbito de convivencia administrativo, y se utilizará la expulsión como tipo de sanción.

\section{MÉTODO}

\section{Participantes}

Se contó con la colaboración de 72 estudiantes, 36 hombres y 36 mujeres elegidos entre aquéllos que se encontraban en el campus universitario y que accedieron voluntariamente a participar en la investigación. La asignación a cada una de las situaciones experimentales se realizó de manera aleatoria con el fin de garantizar la equivalencia de los grupos.

\section{Diseño}

Se recurrió a un diseño de investigación experimental con varios grupos de comparación y mediciones post (Hernández, Fernández y Baptista, 2006). No fue posible incluir mediciones previas al tratamiento, en tanto este hacía parte integral de la situación que permitía registrar la conducta de transgresión.

Tabla 1

Diseño Experimental

\begin{tabular}{cc}
\hline RGe1 & X1 0 \\
\hline RGe2 & X2 0 \\
\hline RGe3 & X3 0 \\
\hline RGe4 & X4 0 \\
\hline
\end{tabular}

Nota: $\mathrm{R}=$ aleatorización; $\mathrm{Ge} n=$ grupo experimental; 0 = medición; $\mathrm{X} n$ = condición experimental.

\section{Procedimiento}

Selección e instrucciones. Después de seleccionar a los sujetos, se explicó el carácter voluntario de su participación en la investigación y, además, se aclaró que existía información sobre el experimento que sólo conocerían al terminar el estudio. Quienes aceptaron participar de la investigación bajo estas condiciones firmaron el consentimiento informado y fueron asignados de manera aleatoria a una de las cuatro situaciones experimentales.
Para todas las condiciones, cada grupo de personas fue situado en un salón amplio que disponía de pupitres para cada participante. Posteriormente, a cada participante se le entregaron las instrucciones y 20 hojas que tenían impresa una cuadricula con 324 casillas, que podían identificarse con una coordenada según el número las filas (del 1 al 27), y la letra de las columnas (de la A a la L) (véase figura 1). Seguidamente se leyeron las siguientes instrucciones (que también se les entregaron por escrito):

"Se le han entregado 20 hojas con una cuadrícula, en cada una de las cuales debe marcar libremente con una $\mathrm{X}$ dos casillas. Si las equis marcadas coinciden con la coordenada al respaldo de la hoja, ganará $\$ 50.000$ pesos (27 dólares aproximadamente). Al final de la sesión usted podrá corroborar si acertó.

Recuerde que durante el experimento está prohibido:

Mirar el respaldo de las hojas de cuadrícula.

Levantar la hoja mientras responde.

Inclinarse sobre el pupitre cubriendo la hoja con el cuerpo.

Mirar las plantillas de los demás.

Hablar con otros participantes o por celular.

Si no sigue estas recomendaciones será descalificado(a) inmediatamente y expulsado(a) de la situación perdiendo el derecho al premio aún si hubiera acertado en la coordenada de alguna de las hojas".

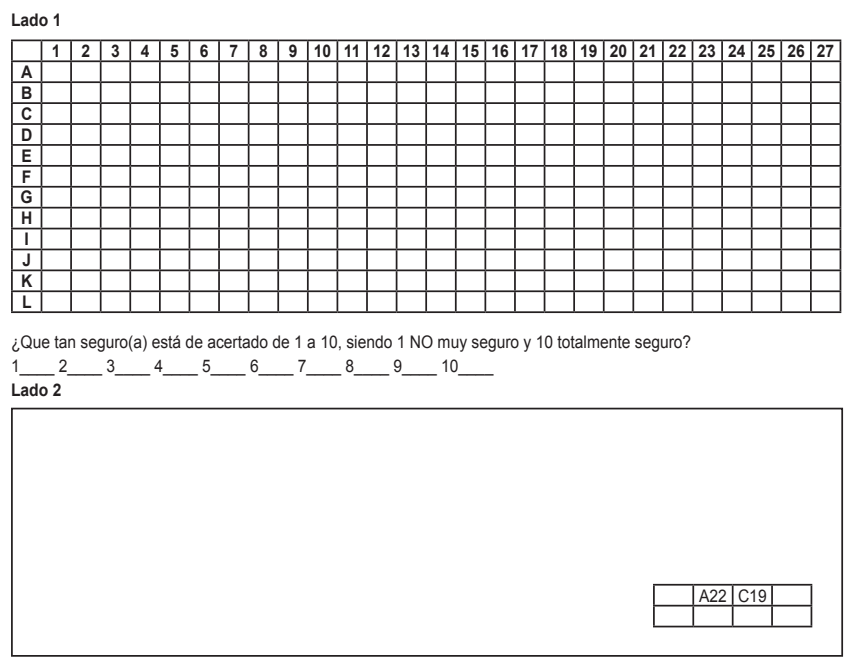

Figura 1. Hoja con cuadrícula y coordenada clave al respaldo.

Al respaldo de cada hoja de cuadrícula, en la parte inferior izquierda, había dos coordenadas (diferentes para cada plantilla) correspondientes a la respuesta correcta (véase Figura 1).

Con el propósito de desviar la atención de los participantes respecto al verdadero propósito del estudio, al final de la cuadricula se incluyó la siguiente pregunta: ¿Qué tan $\operatorname{seguro}(a)$ está de haber acertado de 1 a 10, siendo 1 NO muy seguro 
y 10 totalmente seguro? bajo la pregunta se presentaron 10 opciones de respuesta numeradas (véase figura 1).

Condiciones experimentales. A continuación se describen las cuatro condiciones experimentales aplicadas en el estudio.

RGe1: Sin Autoridad (SA). En esta condición, diez segundos después de que se han dado las instrucciones y entregado el material, un confederado toca a la puerta de la sala y le dice algo al experimentador. Inmediatamente este informa a los participantes que debido a una situación imprevista debe salir durante unos minutos, y les recuerda que deben seguir las recomendaciones, no "hacer trampa" y si alguien termina, debe quedarse en el puesto hasta que el experimentador se lo indique. Luego de esto sale de la sala y sólo entra cuando todos han terminado de responder, lo que se verificaba desde un monitor ubicado en una sala adjunta.

RGe2: Autoridad parcial señalada (APS). Al igual que en la anterior, diez segundos después de que se han dado las instrucciones y entregado el material, una confederada tocó a la puerta y el experimentador inició una animada conversación con ella, dando la espalda a los participantes. Cada 40 segundos el experimentador giraba su cabeza para observarlos durante unos pocos segundos. Esta situación se mantuvo hasta que el 100\% de los participantes terminó de marcar las hojas. En esta condición la autoridad aplica sólo la prescripción y en tanto sólo está presente de manera intermitente, no aplica adecuadamente las otras tres funciones.

RGe3: Instrucciones de autoridad no señalada (IAN). Esta condición es similar a la de SA descrita al principio, con la diferencia de que antes de irse, el experimentador dijo a los participantes que había dos confederados entre los participantes, quienes podrían administrar la sanción en caso de observar una conducta de transgresión. No obstante, esta información era falsa; realmente no había tales confederados.

RGe4: Instrucciones de autoridad no señalada y observación de un caso de sanción (IAN-OS). Finalmente, la cuarta condición es igual a la tercera (las mismas instrucciones e información antes de irse), sólo que ahora realmente existen dos confederados. Uno de ellos actuó como transgresor aproximadamente 40 segundos después de que el experimentador abandonara el lugar, y el otro, actuando como autoridad, inmediatamente administró la sanción de expulsión al transgresor, saliendo junto con él de la actividad. Cabe señalar que la instrucción dada por el experimentador hablaba de dos confederados que sancionarían a los transgresores, por lo que el resto de los participantes suponía que todavía quedaba uno entre ellos, pues desde su perspectiva sólo salió uno con aquél que transgredió la norma.

Las cuatro condiciones experimentales descritas se llevaron a cabo en un salón de $5 \times 7 \mathrm{mts}$., en el que previamente se habían ocultado tres cámaras miniatura en tres bafles del home theater con el que se encuentra equipada la sala, ubicados a los dos lados y al frente de los participantes, a unos $2.50 \mathrm{mts}$. del suelo.

Verificación de Acierto. En esta fase, cuando el 100\% de los participantes de cada situación experimental terminó, se procedió a la comprobación de coincidencias entre las $\mathrm{X}$ marcadas y las coordenadas al respaldo de cada hoja. Durante todo el desarrollo del experimento, sólo una persona acertó sin transgredir las normas, y a ella se le entregó un bono que ocho días después pudo cambiar por cincuenta mil pesos.

\section{Consideraciones éticas}

Todos los participantes firmaron un consentimiento informado, en el cual conocieron de modo muy general el objetivo de la investigación (estudiar las variables que afectan la conducta humana) con el fin de que la información suministrada no generara comportamientos que pudieran alterar los resultados. Sin embargo, en el consentimiento se destacó que el estudio no generaba riesgos asociados ni beneficios directos, que podían abstenerse de participar o retirarse del estudio en cualquier momento, y que había información del estudio que sólo se conocería una vez finalizada la recolección de datos.

Al final del trabajo de campo, vía correo electrónico, se informó a los participantes cuál era el aspecto específico de la conducta que se investigaba y que existían cámaras ocultas en la sala donde se realizó el experimento. Ninguno de los participantes mostró inconformidad o molestia al saberlo.

\section{RESULTADOS}

Los resultados del estudio (véase figura 2) muestran una clara diferencia en la cantidad de transgresiones a la norma entre las diferentes condiciones experimentales.

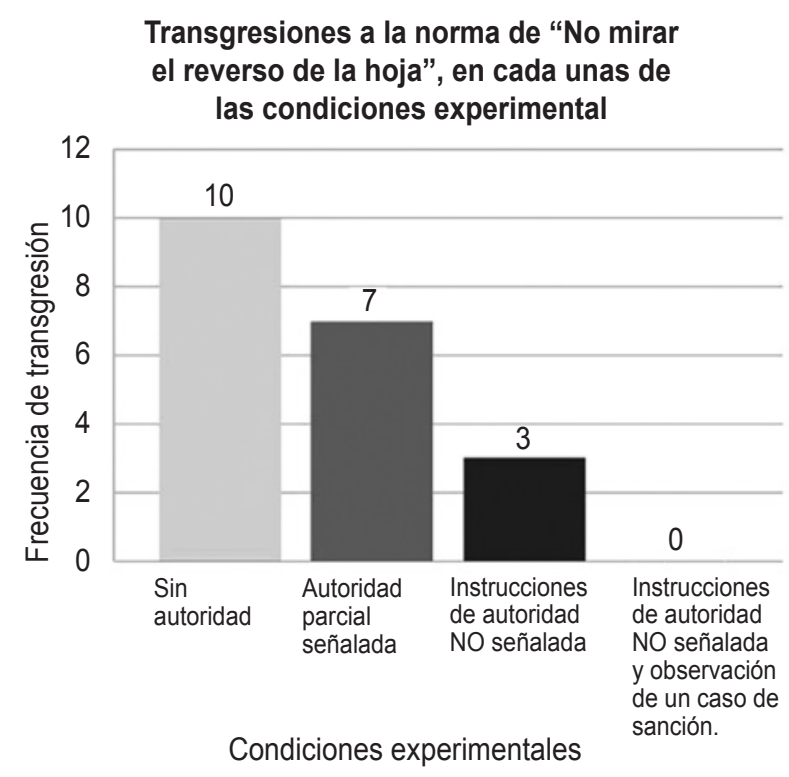

Figura 2.Transgresiones a la norma en las diferentes condiciones experimentales. 
Concretamente, se observa que el $50 \%$ de las transgresiones se presentó en la condición sin autoridad (SA), seguida de autoridad parcial señalada (APS) con el 35\%; luego, con el 15\% de transgresiones, la condición sin autoridade instrucciones de autoridad no señalada (IAN) y, finalmente, la condición de instrucciones de autoridad no señalada y observación de un caso de sanción (IAN-OS), en la que no se presentó ni un solo caso de transgresión ( $0 \%)$. Al aplicar la prueba no paramétrica Kruskal-Wallis, estas diferencias muestran significancia estadística (véase tabla 2).

Tabla 2

Prueba de Kruskal-Wallis para estimar significancia de diferencias entre condiciones experimentales.

\begin{tabular}{|c|c|c|c|}
\hline \multicolumn{4}{|c|}{ Prueba de Kruskal-Wallis } \\
\hline \multicolumn{4}{|c|}{ Rangos } \\
\hline \multirow{6}{*}{$\begin{array}{l}\text { Transgresión a } \\
\text { la norma }\end{array}$} & Condición experimental & $\mathrm{N}$ & Rango promedio \\
\hline & SA & 16 & 49 \\
\hline & APS & 19 & 39,76315789 \\
\hline & IAN & 18 & 32,5 \\
\hline & IAN-OS & 19 & 26,5 \\
\hline & Total & 72 & \\
\hline
\end{tabular}

Transgresión a la norma

$\begin{array}{lc}\text { Chi-cuadrado } & 18,54767206 \\ \text { gl } & 3 \\ \text { Sig. asintót. } & 0,000339055 \\ \begin{array}{l}\text { a. Prueba de Kruskal-Wallis b. Variable de agrupación: Condición } \\ \text { experimental }\end{array}\end{array}$

Nota: $\mathrm{SA}=$ Sin autoridad; $\mathrm{APS}=$ Autoridad parcial señalada; $\mathrm{IAN}=$ Instrucciones de autoridad no señalada; IAN-OS= Instrucciones de autoridad no señalada y observación de un caso de sanción.

Al observar la ocurrencia de transgresiones a la norma en las distintas situaciones según el sexo de los participantes, se nota una diferencia marcada en la condición de autoridad parcial, donde no se observa transgresión en las mujeres (véase figura 3).

De igual manera, se presentan diferencias importantes en la condición SA. Por otra parte, en la condición IAN la diferencia fue sólo de un punto, y en IAN-OS no se observó transgresión en mujeres ni en hombres.

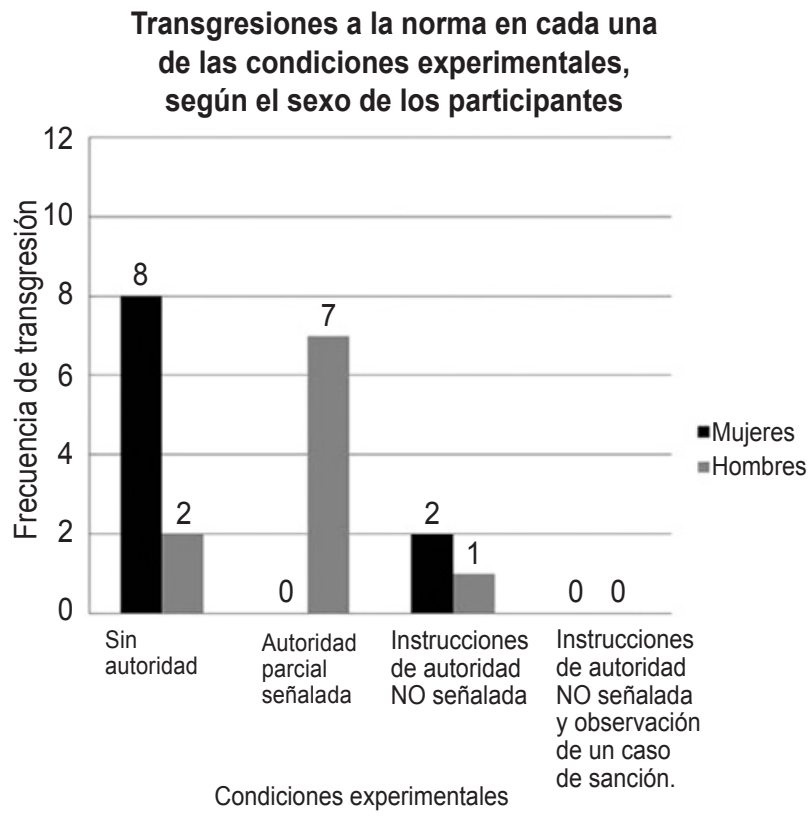

Figura 3. Transgresiones a la norma en las diferentes condiciones experimentales según el sexo de los participantes.

\section{DISCUSIÓN}

Las condiciones experimentales con menos transgresiones a la norma, tanto para hombres como para mujeres, fueron aquéllas con instrucciones de autoridad no señalada. Se nota ausencia total de transgresiones cuando además de estas instrucciones, los participantes observaban un caso de sanción de la transgresión mediante expulsión, administrada por una autoridad que no estaba señalada como tal.

La ocurrencia y observación de una conducta de transgresión que es sancionada por una autoridad inicialmente no identificable, puede interpretarse como una microcontingencia ejemplar, que reguló de manera sustitutiva las interacciones de los sujetos con la condición experimental después de que los confederados salieron de la sala (Rodríguez, 1995). Es evidente que en la situación IAN también se presentó regulación sustitutiva generada por las instrucciones iníciales; sin embargo, en la condición IAN-OS, al parecer la observación adicional de una microcontingencia ejemplar mejoró la efectividad de dicha regulación.

Por otra parte, se resalta que en las dos condiciones con instrucciones de autoridad no señalada, la autoridad fue efectiva aun cuando su poder se basaba en la riqueza (privarlos de la oportunidad de ganar 50.000 pesos al retirarlos del experimento) y sólo se aplicaron contingencias de prescripción y administración de la sanción, pues por su rol en la situación, los confederados no podían aplicar contingencias de supervisión ni de regulación. 
Estos aspectos -dimensión y fuente del poder- se abordaron como objeto central en los estudios de Rangel, Ribes, Valdez y Pulido (2011) y Rangel y Ribes (2009), donde la autoridad por la fuerza y la función de prescripción fueron más efectivas en la ocurrencia de conducta obediente. No obstante, los datos del presente trabajo no confirman ni contradicen dichos resultados, en tanto no se incluyeron variaciones sistemáticas en las contingencias de la dimensión de poder, ni en su fuente de origen.

En cuanto a las diferencias entre hombres y mujeres en las condiciones de SA y APS, aunque el presente estudio no permite sustentar una explicación concreta al respecto, a manera de hipótesis puede pensarse que dichas diferencias estarían relacionadas con diferencias en la historia interactiva individual de los participantes, o posiblemente de hombres y mujeres como grupos. En apoyo a esta última posibilidad, existen algunos planteamientos (Hernández y Pacheco, 2009; Chiale y Husmann, 2008; Rathus y Fichner 2005), que sugieren que es posible que las prácticas convencionales compartidas que constituyen la cultura (Ribes, 1992), hayan establecido metacontingencias diferenciales según el sexo frente la transgresión u obediencia de ciertas normas en circunstancias particulares.

No obstante, a pesar de estas diferencias en la transgresión de normas entre hombres y mujeres, en general, los resultados sugieren que la imposibilidad de discriminar la presencia o ausencia de la autoridad en una situación particular, disminuye la probabilidad de que se presente transgresión de una norma formulada explícitamente. En el caso de que estudios posteriores confirmen estos resultados, se prevén grandes implicaciones prácticas para el diseño de estrategias que optimicen la regulación del comportamiento.

En relación con lo anterior, y aunque diseñadas desde otras perspectivas teóricas, ya se han reportado algunas experiencias satisfactorias sobre problemas reales abordados mediante la aplicación de contingencias que dificultan discriminar la presencia o ausencia de la autoridad. Así, por ejemplo, Mockus (1998) reporta resultados positivos en la convivencia entre peatones y conductores de la ciudad de Bogotá (Colombia) al utilizar, entre otras estrategias, biombos con policías pintados en las caras exteriores y pequeñas ventanas que permitían a quien estuviera adentro mirar hacia las vías. En estos biombos ubicados en lugares estratégicos efectivamente podía estar un policía que administraría una sanción ante una infracción de tránsito o podía estar un ciudadano sin la autoridad competente para hacerlo. No obstante, para un conductor o peatón era bastante difícil discriminar desde la vía si se trataba de un policía real.

Finalmente, aunque para esta investigación no se recurrió al prototipo de la metodología de investigación de la conducta social propuesta por Ribes (2001), se considera que la situación experimental utilizada permite observar aspectos relevantes relacionados con la dimensión funcional de poder, específicamente, la manera en que algunas variables interfieren con la efectividad de una figura de autoridad para impedir la transgresión de normas explicitas.

\section{REFERENCIAS}

Bouzas, A., Orduña, V. \& Palafox, P. G., (2006). Castigo e interacción de respuestas en programas múltiples: Efectos de la localización de la señal. Revista Mexicana de Psicología, 23(1), 69-77.

Carpio, C., Silva, H., Pacheco, P. L., Cantoran, E., Arroyo, R., Canales, C., Pacheco, P. (2008a). Efectos de consecuencias positivas y negativas sobre la conducta altruista. Universitas Psychologica, 7(1), 97-107.

Chiale, G. \& Husmann, G. (2008). La Trampa de los Manipuladores: Cómo identificarlos y aprender a decir Basta. Buenos Aires: Del Nuevo Extremo.

Domjan, M. (2007). Principios de aprendizaje y conducta. Texas: Thomson.

Hernández, R., Fernández, C. \& Baptista, P. (2006). Metodología de la Investigación. México: McGraw-Hill.

Hernández M. R. \& Pacheco, S. B. (2009). Nueva mirada psicológica al "ser mujer: "despertar, transgredir y renacer en el arte. Ciencia y Sociedad, 34(3), 331-34.

Medina, I. F. \& Polanco, L. A. (2009). Efectos de la variación de las instrucciones que señalan relaciones de poder, intercambio y sanción sobre la conducta de cooperación no cooperación. Suma Psicológica, 16(2), 15-25.

Mockus, A. (1998). Armonizar ley, moral y cultura: cultura ciudadana, prioridad de gobierno con resultados en prevención y control de violencia en Bogotá. Recuperado el 20 de julio del sitio web Red Académica: http://www.redacademica.edu.co/archivos/redacademica/proyectos/ddhh/ autoformacion_ddhh/unidad10/anexo_10-3_ley_moral_y_ cultura.pdf

Rangel, B. N. \& Ribes, E. (2009). Level of Authority and Response Cost in the Obebedience of Schoolchildren. Journal of Behavior, Health \& Social Issues, 1(2), 53-65.

Rangel, B. N., Ribes, E., Valdez, V. y Pulido, Á. L. (2011). Las funciones de poder en la obediencia y el cumplimiento de niños escolares. Revista Mexicana de Análisis de la Conducta, 2(37), 205-227.

Rathus, S. \& Fichner, L. (2005). Human Sexuality in a World of Diversity. Massachusetts: Pearson.

Ribes, E. (1992). Factores micro y macro sociales participantes en la regulación del comportamiento psicológico. Revista Mexicana de Análisis de la Conducta, 18(3), 39-55.

Ribes, E. (2001). Funcional dimensions of social behavior: Theoretical considerations and some preliminary data. Revista Mexicana de Análisis de la Conducta, 27(2), 285-306.

Ribes, E. (2007). Estados y límites del campo, medio de contacto y análisis molar del comportamiento: reflexiones teóricas. Acta Comportamentalia, 15(2), 229-259. 
Ribes, E. \& López, F. (1985). Teoría de la conducta: un análisis de campo y paramétrico. México: Trillas.

Ribes, E. \& Rangel, N. (septiembre, 2002). A comparison of choice between individual and shared social contingencies in children and young adults. European Journal of Behaviour Analysis, 3(2), 61-73.

Ribes, E., Rangel, N., Juárez, A., Contreras, S., Abreu, A., Álvarez, A., Casillas, J. (2003). Respuestas sociales forzadas y cambio de preferencia entre contingencias individuales y sociales en niños y adultos. Acta Comportamentalia, 11(2), 197-234.

Ribes, E., Rangel, N. y López V. F. (2008). Análisis teórico de las dimensiones funcionales del comportamiento social. Revista Mexicana de Análisis de la Conducta, 25(1), 45-57.

Ribes, E., Rangel, N., Magaña, C., López, A. \& Zaragoza, A. (2005). Efecto del intercambio diferencial equitativo e inequitativo en la elección de contingencias sociales de altruismo parcial. Acta Comportamentalia, 13(2), 159-179.
Ribes, E., Rangel, N., Magaña, C., Ramírez, E. \& Valdés, U. (2005). Aislamiento verbal y elección de contingencias sociales en altruismo parcial. Avances en la Investigación Cientifica en el CUCBA, 16, 472-477.

Ribes, E., Rangel, N., Zaragoza, A., Magaña, C., Hernández, H., Ramírez, E., Valdez, U. (2006). Effects of differential and shared consequences on choice between individual and social contingencies. European Journal of Behavior Analysis, 7(1), 41-56.

Ribes, E., Rangel, N., Casillas, J. R., Álvarez, A., Gudiño, M., Zaragoza, A., Gudiño, M. (2008). Inequidad y asimetría de las consecuencias en la elección entre contingencias individuales y sociales. Revista Mexicana de Análisis de la Conducta, 29(2), 131-168.

Rodríguez, M. (1995) La dimensión moral de la conducta desde una óptica interconductual. Acta comportamentalia, 3(1), 55-69. 